\title{
User Association, Power Control and Channel Access Schemes for Two-Tier Macro-Femto Networks: CDF of SINR Analysis
}

\author{
Joydev Ghosh*, Akhil Gupta ${ }^{\dagger}$, Huseyin Haci ${ }^{\ddagger}$, Zoltan Jako ${ }^{\ddagger}$ \\ ${ }^{*}$ School of Computer Science and Robotics, National Research Tomsk Polytechnic University, Tomsk, RUSSIA, \\ Email: joydev.ghosh.ece@gmail.com \\ $\dagger$ School of Electronics and Electrical Engineering, Lovely Professional University, Kapurthala, Punjab, India, \\ Email: akhilgupta112001@gmail.com \\ $\ddagger$ Department of Electrical and Electronics, Near East University, Mersin 10, Turkey, Email: \\ huseyin.haci@neu.edu.tr \\ ${ }^{\S}$ BroadBit Hungary Kft, Hungary, Email: zoltan.jako@ broadbit.net
}

\begin{abstract}
In the rapid development of wireless communications Femtocells provide tremendous improvement in coverage and quality of service for users. Macro-Femto based networks are envisioned to be the de-facto solution for providing ultrahigh speed communications in next generation mobile wireless networks. This paper studies two-tier Macro-Femto networks and proposes a collection of novel technologies to address the interference problems. First, a novel user association scheme is proposed that aims to optimize the load among Femto base stations (FBSs). Second, a near-optimal ergodic search algorithm is proposed to regulate the power consumption at Macro base stations (MBSs) and improve energy efficiency. Third, a channel access mechanism is proposed for FBSs that aims to minimize inter-tier interference. For the proposed system, CDF of SINR is derived and used for performance investigation. Simulation results show that the proposed system can significantly outperform a popular, conventional cognitive radio based system for all the considered simulation scenarios.
\end{abstract}

\section{INTRODUCTION}

The telecommunication industry has seen an explosion in a variety of applications and services; such as network gaming, video streaming and social networking as now a days these have become part of peoples' life. Consequently, the amount of mobile devices, the request for data rates and data traffic are continuously increasing. Globally each year, the amount of mobile broadband subscribers is rising by about 25 percent, and it is predicted to be 7.7 billion by 2021 [1] and mobile data to be 351 exabytes by 2025 [2]. Future wireless networks need to be able to convey much more data (the fifth generation $(5 \mathrm{G})$ networks are predicted to convey 1000 times the volume of mobile data compared to the fourth generation (4G) networks) in order to accommodate such number of users with very large data communication requirements channel capacity should be increased drastically. Increasing communication channel bandwidth and signal quality are the two important and desirable properties to improve the channel capacity. The radio frequency spectrum has always been a scarce resource for wireless communication due to being a limited natural resource that can not be fabricated [3]. Thus there has been a lot of research to increase the communication's signal quality. Multiple-Input-Multiple-Output (MIMO), Distributed Antenna Systems (DAS), Device-to-Device (D2D), Cloud Radio Access Networks (C-RAN) and Non-orthogonal Multiple Access (NOMA) are interesting concepts for $5 \mathrm{G}$ and beyond networks that aim to improve signal quality [2]. Another interesting concept that can significantly increase the signal quality is the use of Femtocells [4]. It has been observed that Macrocells alone could not provide the quality of service $(\mathrm{QoS})$ to the indoor user due to the signal's inefficiency to penetrate through the walls. Femtocells are low power base station that converts the centralized cellular networks into distributed networks, typically used for indoor or at the cell edge communication. These can also be understood as a subset of the small cell concept, in which around 4-16 mobile devices can be connected for simultaneous communication. Besides bringing the antenna closer to the user to improve the signal quality, Femtocell can be easily integrated with other interesting signal processing mechanism, such as MIMO, CRAN and NOMA for even better signal quality. This is another important advantage of Femtocell that makes it an interesting open research topic for $5 \mathrm{G}$ and beyond networks. Hence this paper studies Femtocell based networks with their specific properties and related new research dimensions.

The access point of femtocell is known as femto access point (FAP) [5]. It improves coverage, QoS and reduces battery drain due to short range transmissions [6]. FAP is termed as Home Node B (HNB) in $3 \mathrm{G}$ and it is also termed as Home Evolved Node B (HeNB) in Long Term Evolution (LTE) and widely deployed in today's networks. Also it is expected that in $5 \mathrm{G}$, small cells will be employed as primary access points. Femtocells are a strong candidate for small cell deployments in 5G and beyond networks. 5G technologies need to provide highly improved communication data rates and low latency. This paper investigates the opportunity of offloading to Femtocells via user association rules to meet these requirements of future networks. It also articulates on computing the communications efficiency of two-tier networks 
by means of cumulative distribution function (CDF) of users' signal to interference plus noise ratio (SINR). The main contributions of this paper are as follows:

- a novel user association scheme is proposed that optimizes the load among Femto base stations (FBSs) with the goal of improving achieved capacity.

- a near-optimal ergodic search algorithm is developed to regulate the power consumption at Macro base stations (MBSs) and improve energy efficiency by turning-off MBSs whose load can be transferred to FBSs in accordance with the optimization constraints.

- a channel access mechanism for FBS is developed to minimize inter-tier interference by exploiting available channels in a cognitive and opportunistic way.

- the probability mass function (PMF) of a Macro-Femto network with the proposed scheme is derived for performance analysis. Further, the CDF of used channels is obtained from the PMF and then overall CDF of the network is established by using conditional CDF (CCDF) of SINR and PMF of the used channels. Finally, the performance comparison is done between the proposed and popular existing schemes.

The rest of the paper is organized as follows. In section II, related works are discussed. The system model is provided in section III, where we describe and analyze the proposed user association rule based scheme and channel access operation of the Femtocells, respectively. Further, the power control scheme proposed to regulate power consumption at MBSs is given in section III-B. Section IV presents the results and discussion of the proposed scheme, and the conclusion is finally presented in section V. The key notations and their descriptions used in this paper are listed in Table I.

\section{RELATED WORKS}

In 2016, 3GPP standardized the C-V2X (Cellular Vehicle to Everything) [7] to meet the increasing communication needs of the automotive industry. The intelligent services, such as cooperative collision warning and autonomous driving, can only be enabled through reliable communication of data collected by vehicles and roadside infrastructure. Femtocells are proposed as a candidate to provide this reliable wireless communications infrastructure for $\mathrm{C}-\mathrm{V} 2 \mathrm{X}$ standard [8]. A performance comparison for downlink transmissions at a vehicular network containing only MBS and MBS with mobile FBS has been done by [9], [10]. It has been shown that employing FBSs can significantly improve average end-to-end delay and throughput, as well as energy efficiency. Further, a multiobjective optimization problem has been developed by [11] for uplink transmissions at a two-tier Macro-Femto network with mobile end-users. The access control, resource allocation and power management stages are optimized through a multistage decision making process. Significant improvement at system throughput and end-user QoS satisfaction has been shown compared to Macro-only networks. Thus Femtocells are a timely and important research topic and require much more research to show their potential performance gain in downlink and uplink transmissions.
Table I: List of symbols

\begin{tabular}{|c|c|}
\hline Symbol & Description \\
\hline$\Phi$ & $\begin{array}{l}\text { Independent spatial Poisson point process (PPP) } \\
\text { distribution }\end{array}$ \\
\hline$\lambda$ & FBS density per unit area \\
\hline$r_{m}$ & Radius for a circular region of network service \\
\hline $\mathbb{R}^{2}$ & Area of the network service \\
\hline $\bar{P} P_{f}$ & FBS transmit power \\
\hline$P_{m}$ & MBS transmit power \\
\hline $\bar{h}$ & $\begin{array}{l}\text { Small scale fading (SSF) coefficients of the chan- } \\
\text { nel }\end{array}$ \\
\hline$\alpha$ & Path loss exponent \\
\hline$N_{0}$ & Additive white Gaussian noise (AWGN) \\
\hline $\begin{array}{l}B \\
\mathscr{M}_{\text {on }}\end{array}$ & $\begin{array}{l}\text { Bandwidth of the system } \\
\text { Set of turned on BSs }\end{array}$ \\
\hline$N_{f}$ & Number of active UEs \\
\hline$\xi$ & $\begin{array}{l}\text { Design metric in regard to the regulated power } \\
\text { consumption at MBS }\end{array}$ \\
\hline$\varpi$ & Traffic load density \\
\hline$\delta_{i}=0$ & Energy efficiency decider for idle and active states \\
\hline$\eta$ & Network load density \\
\hline$\Psi$ & Degree of load balancing \\
\hline$\nu_{i}$ & BS density vector \\
\hline$q$ & Probability vector \\
\hline$\gamma$ & Signal to interference plus noise ratio (SINR) \\
\hline$C$ & Shannon capacity \\
\hline$\gamma_{t h}$ & Channel detection threshold \\
\hline$\Omega$ & The separation of a user from its linked BS \\
\hline $\mathbb{P}_{\Omega}$ & $\begin{array}{l}\text { The existence probability of the BSs in the distance } \\
D \text { in the context of } \Omega\end{array}$ \\
\hline $\mathbb{F}_{\Omega}(D)$ & $\begin{array}{l}\text { Cummulative distribution function (CDF) in the } \\
\text { context of } \Omega \text { and } D\end{array}$ \\
\hline$\mho_{\Omega}(\Omega)$ & Probability density function (PDF) of $\Omega$ \\
\hline $\mathbb{P}$ & Coverage probability \\
\hline $\mathbb{P}_{X}$ & $\begin{array}{l}\text { Probability mass function (PMF) for the random } \\
\text { variable } X\end{array}$ \\
\hline $\mathbb{F}_{X}$ & CDF for the random variable $X$ \\
\hline$f_{\gamma}(\gamma)$ & $\begin{array}{l}\text { Conditional CDF (CCDF) of SINR using PDF of } \\
\Omega\end{array}$ \\
\hline $\mathbb{F}$ & $\begin{array}{l}\text { Overall CDF of the network using the PMF of the } \\
\text { utilised channels and CCDF of SINR for a user }\end{array}$ \\
\hline
\end{tabular}

Interference management is a crucial aspect in two-tier Macro-Femto networks, since co-tier and inter-tier interference may become a performance limiting factor if not properly dealt with [12], [13]. A cognitive radio (CR) based resource allocation scheme has been proposed by [14] that is shown to maximize the Femtocell throughput by minimizing intertier interference. In [15] a cooperative game theory based approach is taken to minimize interference in cooperative Femtocell networks [16]. It has been shown that throughput, fairness, spectrum spatial reuse and energy efficiency has been improved with the minimization of interference. A particleswarm optimization (PSO) based resource allocation algorithm has been proposed by [17] for interference minimization. When PSO is applied together with iterative waterfilling algorithm, the best results were obtained. Therefore there are many interesting researches proposed for interference management in two-tier Macro-Femto networks with still room for improvement [18]-[20].

Another important design aspect that is unique to two-tier Macro-Femto networks is the type of access mode employed by the Femtocell tier [21]. Femtocell can be implemented in three different access modes classified on the basis of access 


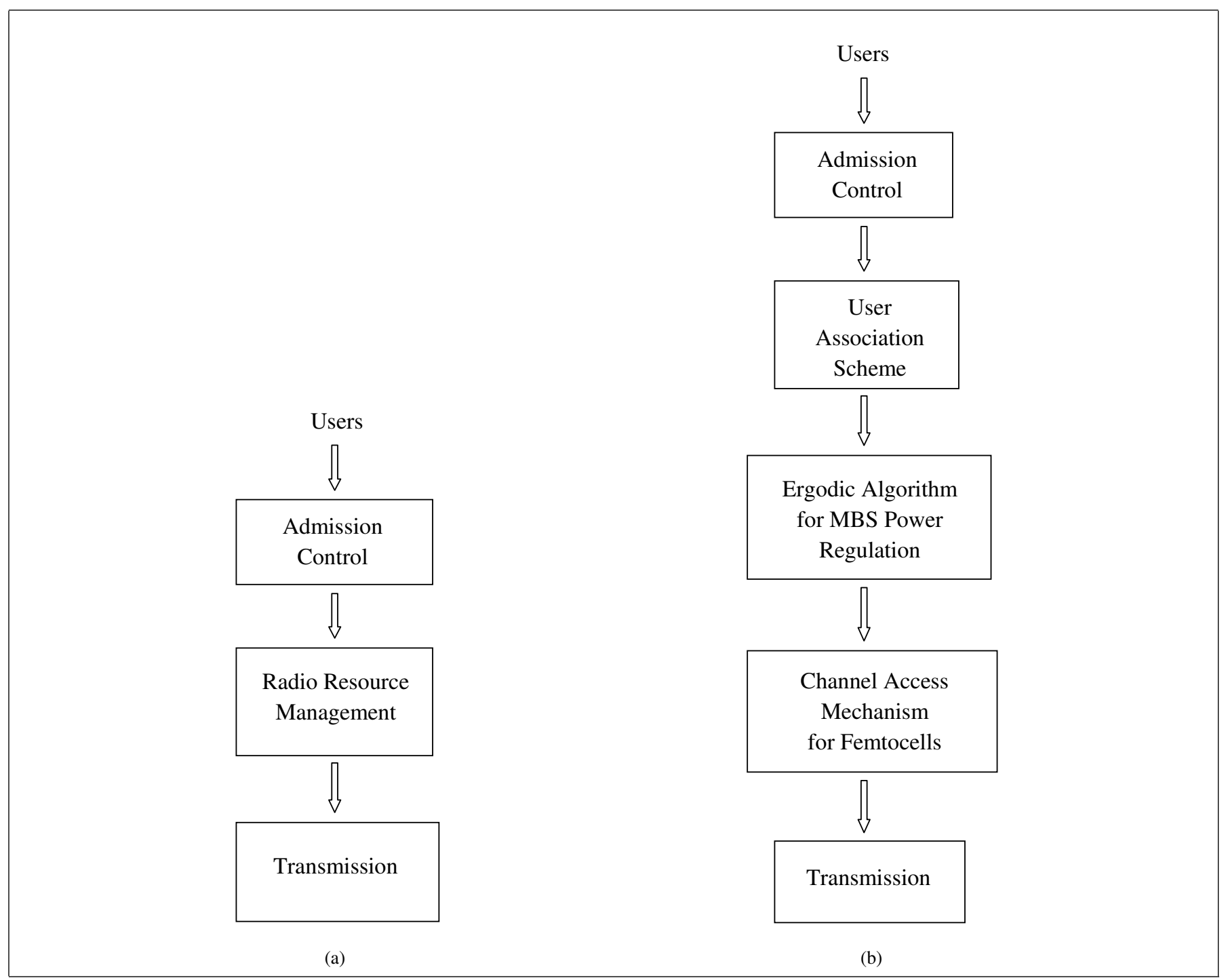

Figure 1: The illustrations of (a) a conventional two-tier Macro-Femtocell network and (b) the proposed system (Fig. 1b).

control, such as open access mode which allows all the users within the vicinity of Femto network to use the femtocell. This mode suffers from increased number of handovers and associated signaling overheads. Closed access mode which allows only predefined users to access the femtocell, the inter-tier interference is the major disadvantage of this mode. And hybrid access mode is a combination of both open and closed access modes [22]. The selection of the appropriate access mode plays an important factor at the network performance. An interference coordination algorithm based on access control in Macro-Femto networks has been proposed by [23]. It has been shown that interference coordination approach operated in hybrid access mode achieved better system performance than closed access mode. [24] considered the uplink transmissions with hybrid access mode and proposed a multilevel optimization method for access control, resource allocation and power management. The results demonstrate significant improvement in system utility compared to existing methods. Another interesting method that demonstrated promising results with various type of access modes is the
Fraction Frequency Reuse (FFR) [25], [26]. A cell sizing based energy optimization method has been proposed for Macro-Femto networks in open access mode by [27]. The results demonstrated improved energy efficiency and interference coordination with the proposed scheme. [28] proposed an interesting power control scheme for blockchain-based femtocell networks. Simulation results show that the proposed scheme improves data transmission rate and guarantees the delay requirement for mobile applications. Other interesting researches in radio resource management (RRM) for Femtocell are [29]-[31].

\section{System Model AND Proposed ApProACH}

A list of symbols used in the paper is given by Table I. The proposed system model is comprised of a MBS facilitating network services for a circular region of radius $r_{m}$ and area $\mathbb{R}^{2}$, and a set of FBSs that are deployed by an independent spatial Poisson point process (PPP) distribution, $\Phi$ with density per unit area given as $\lambda$. Thus, mean number of FBSs can be assumed as $\mathscr{M}=\lambda \times \mathbb{R}^{2}$. In the context of load balancing the 
most important parameter is the traffic load density, denoted by $\varpi$ which can be defined as the packet transfer rate per unit area subject to packet sizes being independently distributed with unity mean. $\varpi$ at location $X$ is finite for $X \in \mathbb{R}^{2}$. This certainly signifies a great deal of liability to vary in spatial traffic and perhaps big packet sizes. Hence, network load density, denoted by $\eta$, can be defined as the ratio of $\varpi$ to Shannon capacity $C . \eta^{1}$ signifies the time taken by $\varpi$ to reach receiver at location $X$ from the $i^{\text {th }}$ BS.

The SINR, at location $X_{k}$ for the $k^{t h}$ user equipment (UE) with respect to the $i^{\text {th }}$ FBS that is $r_{i}$ distance from the origin, can be expressed by:

$$
\gamma_{i, k}=\frac{P_{f} h_{i, k}\left|r_{i}-X_{k}\right|^{-\alpha}}{\sum_{j \in \Psi_{f}} P_{f} h_{j, k}\left|r_{j}-X_{k}\right|^{-\alpha}},
$$

where $P_{f}$ and $P_{m}$ are the transmit power from the Femtocell and Macrocell respectively, $h_{i, k}{ }^{2}, h_{j, k}$ and $h_{g, k}$ represent small scale fading (SSF) coefficients of the channel between $i^{t h}$ FBS and $k^{t h}$ UE, between interfering $j^{t h}$ FBS and $k^{t h}$ UE, and between $g^{t h}$ MBS and $k^{t h}$ UE respectively; $\left|r_{i}-X_{k}\right|^{-\alpha}$, $\left|r_{j}-X_{k}\right|^{-\alpha}$ and $\left|r_{g}-X_{k}\right|^{-\alpha}$ represent large scale path loss (LSPL) between $i^{t h} \mathrm{FBS}$ and $k^{\text {th }} \mathrm{UE}$, between interfering $j^{t h}$ FBS and $k^{t h} \mathrm{UE}$, and between interfering $g^{\text {th }} \mathrm{MBS}$ and $k^{t h}$ UE respectively, where the path loss exponent $\alpha>2$; $I_{f}=\sum_{j \in \Psi_{f}} P_{f} h_{j, k}\left|r_{j}-X_{k}\right|^{-\alpha}$ indicates co-tier interference generated due to other FBSs (i.e., $j \neq i$ ) for communicating on the same resource block group (RBG) allotted to $s^{t h}$ UE, $I_{m}=\sum_{g \in \Psi_{m}} P_{m} h_{g, k}\left|r_{g}-X_{k}\right|^{-\alpha}$ indicates inter-tier interference generated due to the MBSs for communicating on the same RBG allotted to $k^{\text {th }} \mathrm{UE}$ and $N_{0}$ stands for Additive white Gaussian noise (AWGN) and its normalized value is equal to 1 .

A signal of quasi-stationary nature is principally processed in short time frames. This duration is known as a segment when computing SINR of the user. Therefore, the average SINR can be expressed as [36],

$\gamma_{a v g}=10 \log _{2}\left(\frac{1}{T_{s}} \sum_{a=0}^{T_{s}-1} \frac{\sum_{k=1}^{N_{f}} \hat{s}_{a, k}^{2}}{\sum_{k=1}^{N_{f}}\left(\hat{I}_{f, a, k}^{2}+\hat{I}_{m, a, k}^{2}+\hat{n}_{o, a, k}^{2}\right)}\right)$,

where $\hat{s}_{a, k}^{2}, \hat{I}_{f, a, k}^{2}, \hat{I}_{m, a, k}^{2}$, and $\hat{n}_{o, a, k}^{2}$ are used to denote the estimated received signal, co and cross-tier interference and AWGN for the $k^{t h}$ user, respectively. $T_{s}$ denotes the total number of segments in regard to the signal processing duration.

By Shannon capacity ${ }^{3}$ formula, we can express the transmission rate of the system as,

$$
C=B \sum_{i=1}^{\mathscr{M}_{o n}} \sum_{k=1}^{N_{f}} \log _{2}\left(1+\gamma_{i, k}\right),
$$

where $B$ represents the bandwidth of the system, $\mathscr{M}_{\text {on }}$ represents the set of turned on BSs, $N_{f}$ represents number of active UEs.

In order to address problem formulation, we consider a function, denoted by $q_{i}$, to find the possibility that a data transmission at location $X$ is linked with the $i^{t h}$ BS. The set $\mathscr{F}\left(\mathscr{M}_{o n}\right)$ of the attainable BS density $\nu=\left\{\nu_{1}, \cdots \nu_{|\mathscr{M}|}\right\}$ with the corresponding probability vector $q=\left\{q_{1}, \cdots q_{|\mathscr{M}|}\right\}$ for $\mathscr{M}_{\text {on }} \subseteq \mathscr{M}$ can be expressed as below,

$$
\mathscr{F}\left(\mathscr{M}_{\text {on }}\right)=\left\{\nu \mid \nu_{i}=\int_{\mathbb{R}^{2}} \eta_{i} q_{i} d x\right\}, \forall i \in \mathscr{M}_{\text {on }},
$$

Subject to: $0 \leq q_{i} \leq 1, \forall i \in \mathscr{M}_{\text {on }}, \forall x \in \mathbb{R}^{2}, \sum_{i=1}^{\mathscr{M}_{\text {on }}} q_{i}=1$.

Lemma 1: The attainable set $\mathscr{F}\left(\mathscr{M}_{\text {on }}\right)$ is a convex set. Proof: Assume two BS density vectors $\nu_{i}^{1} \in \mathscr{F}\left(\mathscr{M}_{\text {on }}\right)$ and $\nu_{i}^{2} \in \mathscr{F}\left(\mathscr{M}_{\text {on }}\right), \nu_{i}^{1} \neq \nu_{i}^{2}$. Now their corresponding probability vectors can be given as follows: $q_{i}^{1}=\left\{q_{1}^{1}, \cdots q_{\left|\mathscr{M}_{o n}\right|}^{1}\right\}$ and $q_{i}^{2}=\left\{q_{1}^{2}, \cdots q_{\left|\mathscr{M}_{o n}\right|}^{2}\right\}$, which imply $\nu_{i}^{1}=\int \eta_{i} q_{i}^{1} d X$ and $\nu_{i}^{2}=$ $\int \eta_{i} q_{i}^{2} d X \forall i \in \mathscr{M}_{\text {on }}$. With the consideration of $\nu_{i}$ as a convex function of $\nu_{i}^{1}$ and $\nu_{i}^{2}$ for $\vartheta \in[0,1], \nu_{i}$ can be expressed as: $\nu_{i}=\Phi \nu_{i}^{1}+(1-\Phi) \nu_{i}^{2}=\int \eta_{i}\left[\vartheta q_{i}^{1}+(1-\vartheta) q_{i}^{2}\right] d X$ and $\forall i \in$ $\mathscr{M}_{\text {on }}$. Therefore, $q_{i}=\vartheta q_{i}^{1}+(1-\vartheta) q_{i}^{2}$ obeys (3), which implies $\nu$ is attainable and $\mathscr{F}\left(\mathscr{M}_{\text {on }}\right)$ is a convex set respectively.

The illustrations of a conventional system and the proposed system are given in figure 1 . The proposed system have three main building blocks as the user association scheme, ergodic search algorithm for MBS power regulation and channel access mechanism for Femtocells. Detailed explanation of these building blocks are provided at the rest of this section.

\section{A. User Association Rules and Optimum Attainable FBS load:}

The problem formulation of attainable FBS load from the network and UEs' perspective can be given by,

$$
\phi\left(\nu, \mathscr{M}_{\text {on }}\right)=\sum_{i} 2 \nu_{i}-\left(\nu_{i}-1\right)^{1-\Psi},
$$

where $\Psi$ is the metric considered to decide degree of load balancing.

Above (5) gives us insight from the networks and users of perspective. Now, an arbitrarily chosen user with location $X \in \mathbb{R}^{2}$ will receive service from $i^{t h} \mathrm{FBS}$, if and only if the following is true,

$$
i=\arg \max _{i \in \mathscr{M}} C \eta_{i}^{\Psi}, \quad \forall X \in \mathbb{R}^{2} .
$$

Based on the value of $\Psi$, we classified the user association rules into the following cases:

- Case I: At $\Psi=0$, the association rule is completely referring to the user's perspective. This signifies that the 
decision at this point can be made on the SINR level or capacity level with the optimum attainable FBS load equals to $\sum_{i}\left(\nu_{i}+1\right)$.

- Case II: At $\Psi=1$, the user association rule is slowly but surely moving toward network perspective with the increase of $\Psi$. This is ensuring purely traffic load level operation with the optimum attainable FBS load equals to $\sum_{i} 2 \nu_{i}$.

- Case III: For $\Psi \geq 2$, (5) is performing the operation at network load density level. With further increase of $\Psi$ from 1 , it can be noticed that the operation shifts more toward network load density perspective from traffic load perspective.

\section{B. Regulated power consumption at MBS:}

MBSs usually consume high energy at the receiver end regardless of offered loads. In this consequence, the design metric refers to the ratio of an ongoing consumed power of operating a MBS to the total power,

$$
\xi=\frac{\delta_{i} P_{\max _{i}}}{\left[\delta_{i} P_{\max _{i}}+\left(1-\delta_{i}\right) P_{\max _{i}}\right]},
$$

where $P_{\max _{i}}$ denotes the highest regulated power of $i^{t h}$ MBS while it is entirely exploited. The feature of no power consumption, i.e. $\delta_{i}=0$, in the idle state helps to achieve large energy savings at MBS and slowly increases its power consumption level with the increase in its state of the activity. Hence, it is certainly essential to turn off MBSs with less activity jointly with power-efficient UE connection. In this part of the work, we introduce algorithms that improve the energy efficiency (EE) by effectively dealing with the MBS functioning problem ascertaining the cardinality of a BS set that can be disabled.

UE Connection problem (P-UC): For a provided set of enabled MBSs, denoted by $\mathscr{M}_{o n}$, the P-UC can be expressed as,

$$
\mathscr{X}\left(\mathscr{M}_{o n}\right)=\min _{\nu \in \mathscr{F}\left(\mathscr{M}_{o n}\right)} \phi\left(\nu, \mathscr{M}_{o n}\right)+\sum_{\mathscr{M}_{o n}}\left(1-\delta_{i}\right) P_{\max _{i}} .
$$

The above equation is the optimum condition for underlying UE connection.

MBS functioning problem (P-MF): For a provided set of enabled MBSs, denoted by $\mathscr{M}_{o n}$, the P-UC can be expressed as,

$$
\min _{\mathscr{M}_{o n} \subseteq \mathscr{M}} \mathscr{X}\left(\mathscr{M}_{o n}\right)+\sum_{i=\mathscr{M}_{o n}} \delta_{i} P_{\max _{i}}
$$

where, $\mathscr{F}\left(\mathscr{M}_{o n}\right)$ denotes a set for viable load $\nu=$ $\left\{\nu_{1}, \cdots, \nu_{|\mathscr{M}|}\right\}$ while the set of enabled MBS is $\mathscr{M}_{\text {on }} \subseteq \mathscr{M}$.

Remarks: Conflict of interest may occur between (P-UC) and (P-MF). On one side, (P-UC) attempts to enhance the performance level of $\phi\left(\nu, \mathscr{M}_{o n}\right)$ by releasing the traffic load. On the other side, (P-MF) attempts to articulate on fixing the traffic load to be a subset of $\mathscr{M}_{\text {on }}$ in order to minimize $\sum_{i=\mathscr{M}_{o n}} \delta_{i} P_{\max _{i}}$.
(8) is an objective function and its response with $\nu$ is a continuous convex function for constant $\mathscr{M}_{o n}$, whereas it is turn into a discrete non-convex function for variable $\mathscr{M}_{\text {on }}$. Therefore, MBS functioning on regulated power consumption is a challenging case study with $O\left(2^{\mathscr{M}}\right)$ combinatorial analysis, this makes it computationally hard in a high degree to discover an optimum value using brute-force method for a large number of MBSs [32], [33]. This invokes us to introduce algorithms that follow the problem-solving ergodic search (PSES) of making the locally optimal choice at each stage with the intent of finding a global optimum, as follows

PSES-ON algorithm

1.Initialize: $\mathscr{M}_{\text {on }}=\mathscr{M}$

2. while $\mathscr{M}_{\text {on }}=\mathscr{M}$ do

3. Compute:

$\mathscr{W}_{P S E S(\text { on })}(j)=\frac{\mathscr{X}\left(\mathscr{M}_{o n}\right)-\mathscr{X}\left(\mathscr{M}_{o n} \cup j\right)}{\delta_{i} P_{\text {max }_{i}}}, \forall \mathscr{M} / \mathscr{M}_{\text {on }}$

4.Determine MBS:

$j^{*}=\arg \max _{j \in \mathscr{M} / \mathscr{M}_{o n}}\left\{\mathscr{W}_{\text {PSES(on) }}(j)\right\}$

5.if $\mathscr{W}_{P S E S(o n)}\left(j^{*}\right)>1$, then

6. $\mathscr{M}_{\text {on }} \leftarrow \mathscr{M}_{\text {on }} \cup j^{*}$

7. end if

8. end while

PSES-OFF algorithm

1.Initialize: $\mathscr{M}_{o n}=\mathscr{M}$

2. while $\mathscr{M}_{o n}=\mathscr{M}$ do

3. Compute:

$\mathscr{W}_{P S E S(o f f)}(j)=\frac{\mathscr{X}\left(\mathscr{M}_{o n} j\right)-\mathscr{X}\left(\mathscr{M}_{o n}\right)}{\delta_{i} P_{\text {maxi }}}, \forall j \in \mathscr{M}_{o n}$

4.Determine MBS:

$j^{*}=\arg \min _{j \in \mathscr{M}_{o n}}\left\{\mathscr{W}_{P S E S(o f f)}(j)\right\}$

5.if $\mathscr{W}_{P S E S(o f f)}\left(j^{*}\right)<1$, then

6. $\mathscr{M}_{\text {on }} \leftarrow \mathscr{M}-j^{*}$

7. end if

8. end while

Here, we have considered a parameter $\mathscr{W}_{P S E S(o n)}(j)$ which indicates the on-state advantage for the constant consumed power by MBS $j$. The PSES-ON algorithm begins with starting set of MBS, i.e., $M$, and determine the optimum number of disabled MBS through iterative search to have the maximum $\mathscr{W}_{P S E S(\text { on })}$ (step 4). Thereafter, it chooses the MBS that has obtained in the previous step and append to the list of MBS to turn it on subject to the criterion $\mathscr{W}_{P S E S(o n)}\left(j^{*}\right)>1$, else exit.

\section{Channel Access Mechanism by Femtocells:}

An FBS denoted by $F_{i} \in \epsilon_{f}$, where $\epsilon_{f}$ is the set of available FBSs in the system, can be capable of accessing a channel denoted by $n_{i} \in n$, where $n$ is the set of subchannels available in the system, if and only if the received power at 


$$
\mathbb{P}\left\{n_{\text {used }}=y \| n_{m_{1}} \cup n_{m_{2}} \cup n_{m_{3}}|=j, j \leq y,| n_{s} \mid=4\right\}=\sum_{b=y-j}^{y} \mathbb{P}\left\{\left|n_{m_{4}}\right|=b\right\}\left(\begin{array}{c}
b \\
b-(y-j)
\end{array}\right)\left(\frac{j}{|n|}\right)^{b-(y-j)}\left(1-\frac{j}{|n|}\right)^{(y-j)}
$$

$$
\mathbb{P}\left\{n_{\text {used }}=y|| n_{s} \mid=4\right\}=\sum_{j=0}^{y} \Lambda_{3}(y) \sum_{b=y-j}^{y} \mathbb{P}\left\{\left|n_{m_{4}}\right|=b\right\}\left(\begin{array}{c}
b \\
b-(y-j)
\end{array}\right)\left(\frac{j}{|n|}\right)^{b-(y-j)}\left(1-\frac{j}{|n|}\right)^{(y-j)}
$$

where, $\Lambda_{3}(y)=\mathbb{P}\left\{n_{\text {used }}=y|| n_{s} \mid=3\right\}=\sum_{j=0}^{y} \Lambda_{2}(y) \sum_{b=y-j}^{y} \mathbb{P}\left\{\left|n_{m_{3}}\right|=b\right\}\left(\begin{array}{c}b \\ b-(y-j)\end{array}\right)\left(\frac{j}{|n|}\right)^{b-(y-j)}\left(1-\frac{j}{|n|}\right)^{(y-j)}$

and, $\Lambda_{2}(y)=\mathbb{P}\left\{n_{\text {used }}=y|| n_{s} \mid=2\right\}=\sum_{j=0}^{y} \mathbb{P}\left\{\left|n_{m_{1}}\right|=j\right\} \sum_{b=y-j}^{y} \mathbb{P}\left\{\left|n_{m_{2}}\right|=b\right\}\left(\begin{array}{c}b \\ b-(y-j)\end{array}\right)\left(\frac{j}{|n|}\right)^{b-(y-j)}\left(1-\frac{j}{|n|}\right)^{(y-j)}$

$F_{i}$ from transmitter MBS denoted by $M_{i} \in \epsilon_{m}$, where $\epsilon_{m}$ is the set of available MBSs in the system, is higher than the channel detection threshold denoted by $\gamma_{t h}$ subject to the assumption that all the FBSs are cognitive in nature and performing opportunistic channel access. The channel access operation is performed with the assumption that $F_{i}$ should not reutilize the channel which is already utilized by $M_{i}$, where $M_{i}$ specifically belong to the adjacent MBSs of $F_{i}$. As $\gamma_{t h}$ is becoming a design metric to develop a coexisting network model of FBSs with MBSs, the susceptibility of the network model in spatial domain gets higher with the decrease of $\gamma_{t h}$ which in turn produces a small inter-tier interference and helps in the execution of dynamic reutilization of spatial spectrum, likewise it has reverse effect with the increase of $\gamma_{t h}$.

Let, $\left|n_{s}\right| \sim(\lambda)$, where $\lambda=\pi \rho_{m b s}\left(\frac{P_{m}}{\mu_{m} \gamma_{t h}}\right)^{\left(\frac{2}{\alpha}\right)} \Gamma\left(1+\frac{2}{\alpha}\right)$, |.| indicates number of elements of the set, $\rho_{m b s}$ denotes MBS density, channel gain between a user and an MBS is exponentially distributed with mean $\frac{1}{\mu_{m}}$. If $n_{f t}$ denotes a set of channels utilised by $F_{i}$ at a certain time instant, then: $n_{f t}=\frac{n}{\left(\cup_{\left.m_{t} \in n_{s t} n_{m_{t}}\right)}\right.}$, where $n_{s t}=M_{j} \mid P_{m} h_{m j}\left\|F_{i}-M_{j}\right\|^{-\alpha}$ indicates set of adjacent MBSs to $F_{i}$ and $h_{m j}$ denotes channel gain between a user and an MBS at $j^{t h}$ channel.

Lemma 2: If $n_{\text {used }}$ denotes the number of utilised channels out of $|n|$ available channels within the channel detection region of a referenced FBS positioned at $F_{i} \in \mathbb{R}^{2}$, then its probability mass function (PMF) can be expressed by (10) and (11), respectively.

$$
\begin{aligned}
\mathbb{P}\left\{n_{\text {used }}=y\right\} & =\mathbb{A}\{y=0\} \mathbb{P}\left\{\left|n_{s}\right|=0\right\} \\
& +\sum_{a=1}^{\infty} \mathbb{P}\left\{\left|n_{s}\right|=a\right\} \Lambda_{n}(y), 0 \leq y \leq|n|
\end{aligned}
$$

where $\mathbb{A}\{y=0\}$ is a binary indicator that produces 1 if the condition is satisfied, else 0; $\Lambda_{n}(y)=\sum_{j=0}^{y} \Lambda_{(n-1)}(j) \sum_{z=y-j}^{y} \mathbb{P}\left\{X_{p u}=\right.$ $z\} w\left(z, z-(y-j), \frac{j}{|n|}\right)$, where $w(a, b, c)=\left(\begin{array}{c}a \\ b\end{array}\right) c^{b}(1-c)^{(a-b)}$ and $\Lambda_{1}(j)=\mathbb{P}\left\{X_{p u}=j\right\}$

Proof: Here, an objective is to establish PMF on the distribution of utilised channels within the channel detection region around a referenced FBS. The utilization probability of $y$ channels keeping the limitation of utmost 4 MBS belonging to the channel detection region can be expressed as in (10). Without any limitation on the number of utilised channels within the channel detection region, we get (11). Hence, the lemma can be proved using the above equations.

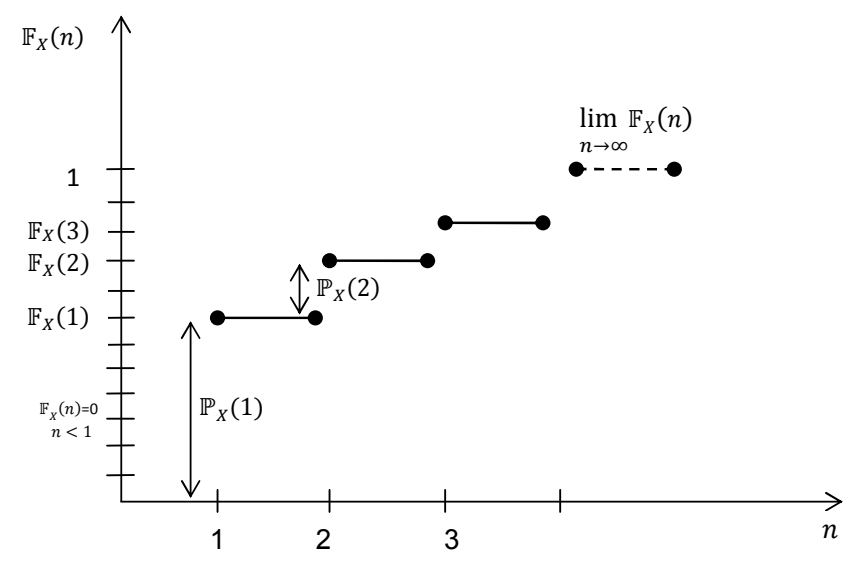

Figure 2: CDF of the random variable given in the problem.

\section{Cumulative Distribution Function (CDF) Analysis}

The CDF in figure 2 illustrates the distribution of a discrete random variable. Especially, we can determine the PMF by 


$$
\begin{aligned}
f_{\gamma}(\gamma) & =\mathbb{E}_{\Omega}\left[\mathbb{P}\left[\gamma_{i, k} \leq \gamma_{t h} \mid \Omega\right]\right] \\
& =1-\mathbb{E}_{\Omega}\left[\mathbb{P}\left[\gamma_{i, k}>\gamma_{t h} \mid \Omega\right]\right] \\
& =1-\int_{\Gamma \geq 0} \mathbb{P}\left[\gamma_{i, k}>\gamma_{t h} \mid \Omega\right] f_{\Omega}(\Omega) d \Omega \\
& \stackrel{(\mathrm{b})}{=} 1-\int_{\Gamma \geq 0} \mathbb{P}\left[\frac{P_{f} h_{i, k} \Omega^{-\alpha}}{\sum_{j \in \Psi_{f}} P_{f} h_{j, k}\left|r_{j}-X_{k}\right|^{-\alpha}+\sum_{g \in \Psi_{m}} P_{m} h_{g, k}\left|r_{g}-X_{k}\right|^{-\alpha}+N_{0}}>\gamma_{t h} \mid \Omega\right] 2 \pi \lambda \Omega \exp \left(-\lambda \pi \Omega^{2}\right) d \Omega \\
& =1-\int_{\Gamma \geq 0} \exp \left(-\lambda \pi \Omega^{2}\right) \mathbb{P}\left[h_{i, k}>\gamma_{t h} P_{f}^{-1} \Omega^{\alpha}\left(I_{f}+I_{m}+N_{0}\right) \mid \Omega\right] 2 \pi \lambda \Omega d \Omega \\
& \stackrel{(\mathrm{c})}{=} 1-\int_{\Gamma \geq 0} e^{-\left(\lambda \pi \Omega^{2}\right)} e^{-\left(\mu \gamma_{t h} P_{f}^{-1} \Omega^{\alpha} N_{0}\right)} \mathscr{L}_{I_{f}}\left(\mu \gamma_{t h} P_{f}^{-1} \Omega^{\alpha}\right) \mathscr{L}_{I_{m}}\left(\mu \gamma_{t h} P_{f}^{-1} \Omega^{\alpha}\right) 2 \pi \lambda \Omega d \Omega \\
& \left.\stackrel{(\mathrm{d})}{=} 1-\int_{\Gamma \geq 0} 2 \pi \lambda \Omega \exp \left(\lambda \pi \Omega^{2}\right) \exp \left(-\mu \gamma_{t h} P_{f}^{-1} \Omega^{\alpha} N_{0}\right)\right) \exp \left(-2 \pi \lambda\left[\int_{\Omega}^{\infty}\left(1-\frac{1}{1+s P_{f} \omega_{f}^{-\alpha}}\right) \omega_{j} d \omega_{j}\right.\right. \\
& \left.\left.\quad+\int_{\Omega}^{\infty}\left(1-\frac{1}{1+s P_{m} \omega_{g}^{-\alpha}}\right) \omega_{g} d \omega_{g}\right]\right) d \Omega .
\end{aligned}
$$

determining the height of the jumps in the CDF function. On the other side, the CDF of a discrete random variable is a step function with left-closed and right-open intervals. With the assumption of $X=n_{\text {used }}$ as the random variable for the range $R_{X}=\left\{n_{1}, n_{2}, n_{3}, \cdots\right\}$ where $n_{1}<n_{2}<n_{3} \cdots,{ }^{4}$ the CDF can be determined from its PMF as follows, let

$$
\mathbb{F}_{X}(n)=\sum_{n_{s} \leq n} \mathbb{P}_{X}\left(n_{s}\right)
$$

Now,

For $n<1, \mathbb{F}_{X}(n)=0$.

For $1 \leq n<2, \mathbb{F}_{X}(n)=\mathbb{P}_{X}(1)$.

For $2 \leq n<3, \mathbb{F}_{X}(n)=\mathbb{P}_{X}(1)+\mathbb{P}_{X}(2)$

The distance $\left|r_{i}-X_{k}\right|$ is a key parameter as it indicates the separation of a user from its linked BS. In general most of the users are served by the nearest BS, thus no interfering BS can be closer than $\Omega=\left|r_{i}-X_{k}\right|$. The existence probability of the BSs in the distance $D$ can be expressed as [34, refer to section 3.4],

$$
\mathbb{P}_{\Omega}\{\Omega>D\}=\exp \left(-\lambda \pi D^{2}\right)
$$

where $D=\min \left\{\left|r_{j}-X_{k}\right|,\left|r_{g}-X_{k}\right|\right\}$. Therefore, the CDF is

$$
\mathbb{F}_{\Omega}(D)=\mathbb{P}_{\Omega}\{\Omega \leq D\}=1-\exp \left(-\lambda \pi D^{2}\right)
$$

and the probability density function (PDF) can be found as,

$$
f_{\Omega}(\Omega)=\frac{d \mathbb{F}_{\Omega}(\Omega)}{d \Omega}=2 \pi \lambda \Omega \exp \left(-\lambda \pi \Omega^{2}\right), \quad \Omega \geq 0
$$

4

Property: For $n_{1} \leq n_{2}, \mathbb{P}_{X}\left(n_{1}<n_{s} \leq n_{2}\right)=\mathbb{F}_{X}\left(n_{2}\right)-\mathbb{F}_{X}\left(n_{1}\right)$. Proof : $\mathbb{P}_{X}\left(n_{s} \leq n_{2}\right)=\mathbb{P}_{X}\left(n_{s} \leq n_{1}\right)+\mathbb{P}_{X}\left(n_{1}<n_{s} \leq n_{2}\right)$. Thus, $\mathbb{F}_{X}\left(n_{2}\right)=\overline{\mathbb{F}}_{X}\left(n_{1}\right)+\mathbb{P}_{X}\left(n_{1}<n_{s} \leq n_{2}\right)$
Applying user association rule on the nearest BS at $\Omega$ from the referenced user, the coverage probability can be written as,

$$
\begin{aligned}
& \mathbb{P}\left[h_{i, k}>\gamma_{t h} P_{f}^{-1} \Omega^{\alpha}\left(I_{f}+I_{m}+N_{0}\right) \mid \Omega\right] \\
& =\mathbb{E}_{I}\left[\mathbb{P}\left[h_{i, k}>\gamma_{t h} P_{f}^{-1} \Omega^{\alpha}\left(I_{f}+I_{m}+N_{0}\right) \mid \Omega, I_{f}, I_{m}\right]\right] \\
& \stackrel{(\mathrm{a})}{=} \mathbb{E}_{I}\left[\mathbb{P}\left[\exp \left(-\mu \gamma_{t h} P_{f}^{-1} \Omega^{\alpha}\left(I_{f}+I_{m}+N_{0}\right)\right) \mid \Omega\right]\right] \\
& =e^{-\left(\mu \gamma_{t h} P_{f}^{-1} \Omega^{\alpha} N_{0}\right)} \mathscr{L}_{I_{f}}\left(\mu \gamma_{t h} P_{f}^{-1} \Omega^{\alpha}\right) \mathscr{L}_{I_{m}}\left(\mu \gamma_{t h} P_{f}^{-1} \Omega^{\alpha}\right),
\end{aligned}
$$

where $(a)$ follows from the fact that $h_{i, k} \sim \exp (\mu), \mathscr{L}_{I_{f}}($. and $\mathscr{L}_{I_{m}}($.$) are the Laplace transform of the cumulative co-$ and inter-tier interferences, respectively, when the randomly chosen mobile user is being served by the Femtocell.

The solution for Laplace transform of $L_{I f}(s)$ is given as

$$
\begin{aligned}
\mathscr{L}_{I_{f}}(s) & =\mathbb{E}_{I_{f}}\left[e^{-s I_{f}}\right] \\
& =\mathbb{E}_{\Phi, h_{j}}\left[\exp \left(-s \sum_{j \in \Psi_{f}} P_{f} h_{j, k}\left|r_{j}-X_{k}\right|^{-\alpha}\right)\right] \\
& =\mathbb{E}_{\Phi, h_{j}}\left[\prod_{j \in \Psi_{f}} \exp \left(-s P_{f} h_{j, k}\left|r_{j}-X_{k}\right|^{-\alpha}\right)\right] \\
& \stackrel{(\mathrm{e})}{=} \mathbb{E}_{\Phi}\left[\prod_{j \in \Psi_{f}} \mathbb{E}_{j_{j}}\left[\exp \left(-s P_{f} h_{j, k}\left|r_{j}-X_{k}\right|^{-\alpha}\right)\right]\right] \\
& \stackrel{(\mathrm{f})}{=} \exp \left(-2 \pi \lambda \int_{\Omega}^{\infty}(1-\right. \\
& \left.\left.\mathbb{E}_{j}\left[\exp \left(-s P_{f} h_{j, k} \omega_{j}^{-\alpha}\right)\right]\right) \omega_{j} d \omega_{j}\right) \\
& =\exp \left(-2 \pi \lambda \int_{\Omega}^{\infty}\left(1-\frac{1}{1+s P_{f} \omega_{f}^{-\alpha}}\right) \omega_{j} d \omega_{j}\right),
\end{aligned}
$$


where $\omega_{j}=\left|r_{j}-X_{k}\right|$, (e) follows from the fact of independent and identically distributed (i.i.d) $h_{j, k}$ and its further independence from independent spatial PPP distribution $\Phi$, and $(f)$ follows from the fact that $\mathbb{E}\left[\prod_{x \in \Phi} f(x)\right]=$ $\exp \left(-\lambda \int_{\mathbb{R}^{2}}(1-f(x)) d x\right)$ for the function $f(x)$. By following (1) with threshold constraint $\gamma_{t h}$, the conditional CDF (CCDF) of SINR using the PDF of $\Omega$ is presented in (17), where (b) follows from the distribution of $f_{\Omega}(\Omega)$ given in (15), $\gamma_{t h}$ indicates the threshold, $I_{f}=\sum_{j \in \Psi_{f}} P_{f} h_{j, k}\left|r_{j}-X_{k}\right|^{-\alpha}$, $I_{m}=\sum_{g \in \Psi_{m}} P_{m} h_{g, k}\left|r_{g}-X_{k}\right|^{-\alpha}$, (c) follows by substituting (16) into (17), and $(d)$ follows by solving the Laplace transform for $L_{I f}(s)$ and $L_{I m}(s)$, given by (18) and (19) respectively.

Similarly, the solution for Laplace transform of $L_{I m}(s)$ is given as

$$
\begin{aligned}
\mathscr{L}_{I_{m}}(s) & =\mathbb{E}_{I_{m}}\left[e^{-s I_{m}}\right] \\
& =\exp \left(-2 \pi \lambda \int_{\Omega}^{\infty}\left(1-\frac{1}{1+s P_{m} \omega_{g}^{-\alpha}}\right) \omega_{g} d \omega_{g}\right) .
\end{aligned}
$$

Using the PMF of the utilised channels and CCDF of SINR for a user, overall CDF of the network can be expressed as below,

$$
\mathbb{F}=\sum_{n \leq n_{s}} \mathbb{P}_{X}\left(n_{s}\right) f_{\gamma}(\gamma)
$$

\section{Results And Discussion}

The proposed schemes can be used in both low and high SINR regions. It should be noted that instantaneous SINR values for users given by (1) may change depending on the dynamic channel conditions and geographical position of the user. The average SINR region for a simulated scenario can be calculated by (2). CDF is a critical parameter for determining the average performance of the network. It is the probability that the function value will lie below certain threshold. It gives the clear picture of the number of outage users even if few UEs get maximum SINR. Also in case of maximum number of FBSs, the traffic on Macrocell gets minimum and number of user in each Femtocell are reduced simultaneously. We have considered fixed number of Femtocells that are randomly located within a given region and UEs are also allocated within the radius of Femtocell. The path loss is evaluated for each UE within or outside the Femtocell coverage, if an UE is within the Femtocell coverage region it will be considered as a service user, i.e. user with desired signal, otherwise it will be considered as an interfering user. Thereafter, SINR of each UE is computed. The simulation network parameters are given by Table II. Network bandwidth of $100 \mathrm{MHz}$ is chosen since such bandwidth can be achieved with LTE-Advance by channel aggregation and it is envisioned that in $5 \mathrm{G}$ networks Femtocells may have $100 \mathrm{MHz}$ bandwidth to support ultra high-speed communications [35].

Figure 3 compares the CDF of SINR performance for a popular cognitive radio based resource allocation scheme developed by [13] and the proposed scheme with various radius of Femtocells. It can be seen from the figure that the proposed scheme outperforms [13] for all the considered
Table II: Network Parameters

\begin{tabular}{|l|l|}
\hline Parameter & Value \\
\hline Network bandwidth & $100 \mathrm{MHz}$ \\
\hline Sub-channels & 512 \\
\hline MBS Tx power (max.) & $43 \mathrm{dBm}$ \\
\hline FBS Tx power & $\begin{array}{l}20 \mathrm{dBm} \text { (max.) } \\
\text { and 0dBm (min.) }\end{array}$ \\
\hline Macro cell radius & $1000 \mathrm{~m}$ \\
\hline Femto cell radius & $<100 \mathrm{~m}$ \\
\hline Antenna pattern for BS of macrocell & Omnidirectional \\
and femtocell & \\
\hline External walls, $W_{e x}$ & $15 \mathrm{~dB}$ \\
\hline Internal walls, $W_{i n}$ & $10 \mathrm{~dB}$ \\
\hline Macrocell Path Loss & 128.1 \\
& $37.6 l o g ~ 10\left(r_{m}\right)+$ \\
& $W_{e x}+W_{i n}$ \\
\hline
\end{tabular}

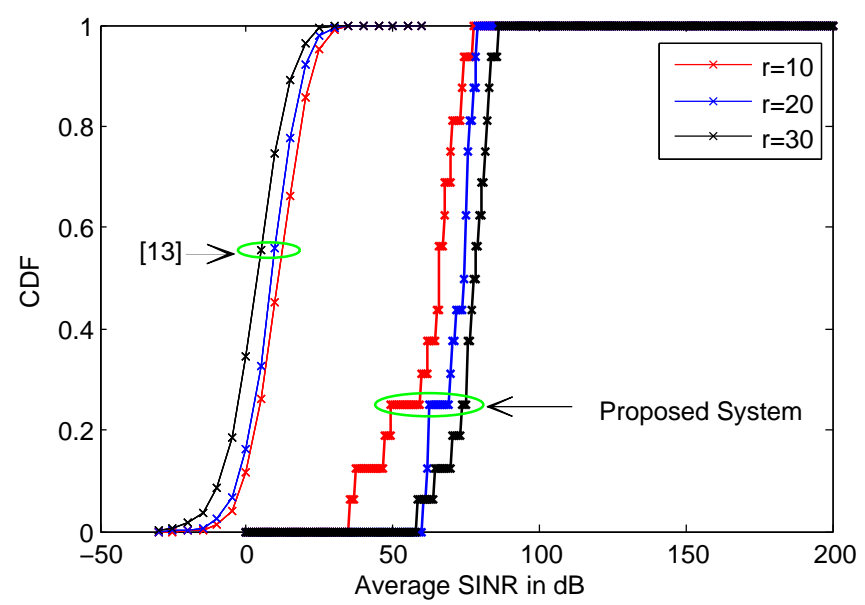

Figure 3: CDF vs SINR for different radius of femtocell with $\mathrm{UE}=1$ in each femtocell.

Femtocell radius. This is due to the ergodic search algorithm developed to regulate power at MBSs to minimize intertier interference from Macro-to-Femto tier. For the proposed scheme, the performance increases as the radius increase since more users can be associated with Femtocells and accordingly more MBSs can be turned-off.

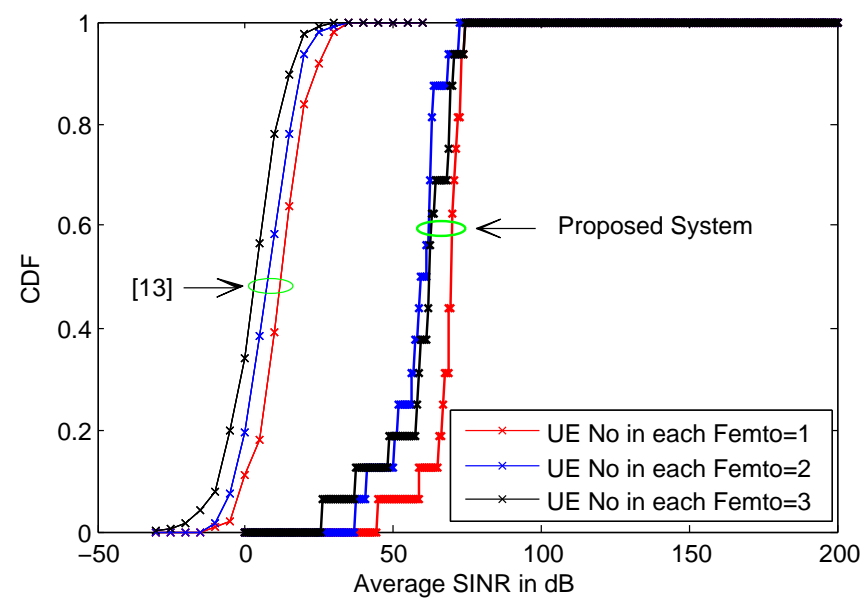

Figure 4: CDF vs SINR for different number of UEs in each femtocell of radius $=10$. 


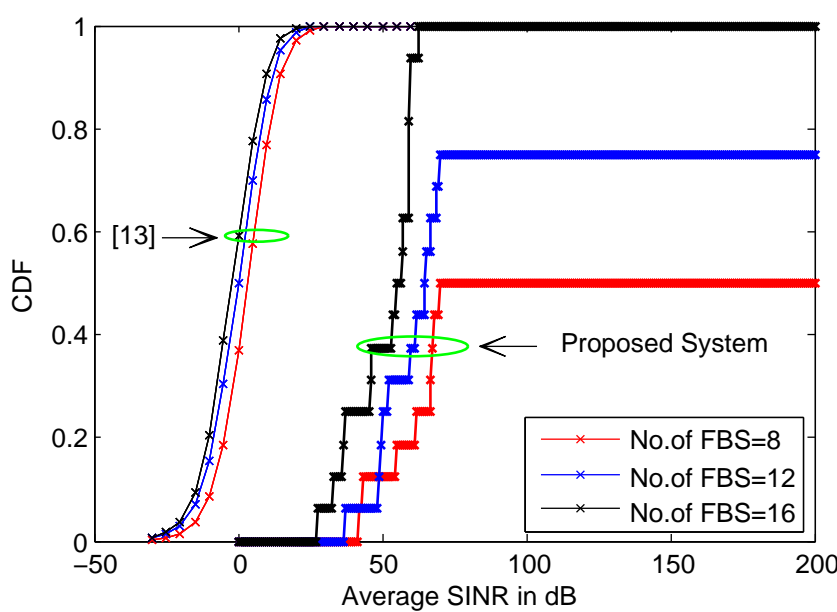

Figure 5: CDF vs SINR for different number of FBSs in the networks.

Figure 4 compares the CDF of SINR performance for [13] and the proposed scheme when the number of users in each Femtocell increases. This is an interesting performance metric since $5 \mathrm{G}$ and beyond networks are expected to have many users connected to Femtocells. The proposed scheme demonstrated significantly better performance compared to [13] for all the considered scenarios.

Figure 5 compares the CDF of SINR performance for [13] and the proposed scheme for various number of FBS. It can be seen from the figure that the proposed scheme outperforms [13] for all the considered cases. The performance of proposed scheme increases as the number of FBSs increases. This is due to the benefit of the proposed user association rule that associates users with best possible FBS to maximize the capacity and help to minimize co-tier interference.

\section{CONClusion}

This paper studies two-tier Macro-Femto networks and proposes a collection of novel technologies to address the interference problems. First, a novel user association scheme is proposed that aims to optimize the load among FBSs and improve achievable capacity. Second, a near-optimal ergodic search algorithm is proposed to regulate the power consumption at MBSs. The aim of the algorithm is to improve energy efficiency by turning-off MBSs whose load can be transferred to FBSs in accordance with the optimization constraints. Third, a channel access mechanism is proposed for FBSs that aims to minimize inter-tier interference by exploiting available channels in a cognitive and opportunistic way. For the proposed system, CDF of SINR is derived and used for performance investigation. It has been shown that the proposed system can significantly outperform a popular, conventional cognitive radio based system for all the considered simulation scenarios.

\section{REFERENCES}

[1] Ericsson, "Ericsson Mobility Report". 2016. [Online]. Available http://www.ericsson.com/res/docs/2015/mobility-report/ ericsson-mobility-report-nov-2015.pdf. Accessed 26 March 2016.
[2] M. Peng, Y. Li, Z. Zhao, C. Wang, "System architecture and key technologies for $5 \mathrm{G}$ heterogeneous cloud radio access networks," IEEE Network, vol. 29, no. 2, pp.6-14, 2015.

[3] J. Ghosh, D. N. K. Jayakody, M. Qaraqe, T. A. Tsiftsis, "Coverage Probability Analytics by Fractional Frequency Reuse Scheme," ITELCON 2017 (Springer Lecture Notes in Electrical Engineering), Istanbul, Turkey, Dec.' 2017.

[4] J. Ghosh, D. N. K. Jayakody, "Game Theoretic Frequency Reuse Approach in OFDMA Femtocell Networks," Transactions on Emerging Telecommunications Technologies, vol. 29, no. 10, June 2018.

[5] V. Chandrasekhar, V. Andrews, V. Gatherer, "Femtocell networks: A survey," IEEE Commun. Mag., vol. 46, no. 9, pp. 59-67, Sep. 2008.

[6] A. AlAmmouri, J. G. Andrews, F. Baccelli, "SINR and Throughput of Dense Cellular Networks With Stretched Exponential Path Loss," IEEE Transactions on Wireless Communications, vol. 17, no. 2, pp. 1147-1160, 2018.

[7] Z. Liu, Y. Xie, K. Y. Chan, K. Ma and X. Guan, "Chance-Constrained Optimization in D2D-Based Vehicular Communication Network," IEEE Transactions on Vehicular Technology, vol. 68, no. 5, pp. 5045-5058, May 2019.

[8] Z. Jako, J. Ghosh,"Network throughput and outage analysis in a poisson and matrn cluster based LTEadvanced small cell networks,"Int. J. Electron. Commun., Vol. 75, pp. 46-52, May 2017.

[9] M. Patra, R. Thakur, C. S. R. Murthy, "Improving Delay and Energy Efficiency of Vehicular Networks Using Mobile Femto Access Points,' IEEE Transactions on Vehicular Technology, vol. 66, no. 2, pp. 14961505, Feb. 2017

[10] T. Mao, G. Feng, L. Liang, S. Qin, B. Wu, "Distributed Energy-Efficient Power Control for Macro-Femto Networks," IEEE Transactions on Vehicular Technology, vol. 65, no. 2, pp. 718-731, Feb. 2016.

[11] B. Niu, V. W. S. Wong, "Network Configuration for Two-Tier Macro-Femto Systems With Hybrid Access," IEEE Transactions on Vehicular Technology, vol. 65, no. 4, pp. 2528-2543, April 2016.

[12] S. A. Khwandah, J. P. Cosmas, Z. D. Zaharis, P. I. Lazaridis, I. A. Glover and S. M. Saleh, "Interference Management Scheme for Cochannel Femtocells," 24th International Conference on Automation and Computing (ICAC), Newcastle upon Tyne, United Kingdom, 2018, pp. $1-4$.

[13] V. Asghari, A. Ghrayeb, S. Affes, "Opportunistic Open-Access MacroFemto Networks with Femto Base Station Selection," IEEE International Conference on Ubiquitous Wireless Broadband (ICUWB), Montreal, QC, 2015, pp. 1-5, 2015.

[14] D. C. Oh, Y. H. Lee, "Cognitive Radio Based Resource Allocation in Femtocell," Journal of Communications and Networks, vol.14, no.3, June 2012

[15] R. Langar, S. Secci, R. Boutaba, G. Pujolle, "An Operations Research Game Approach for Resource and Power Allocation in Cooperative Femtocell Networks," IEEE Transactions on Mobile Computing, vol. 14, no. 4, pp. 675-687, 2015.

[16] B. U. Kazi, G. A. Wainer, "Next generation wireless cellular networks: ultra-dense multi-tier and multi-cell cooperation perspective," Wireless Networks, July 2018

[17] J. Yu, S. Han, X. Li, "A Robust Game-Based Algorithm for Downlink Joint Resource Allocation in Hierarchical OFDMA Femtocell Network System," IEEE Transactions on Systems, Man, and Cybernetics: Systems, vol. 50, no. 7, pp. 2445-2455, July 2020.

[18] H. Haci, H. Zhu, J. Wang,"Performance of Non-orthogonal Multiple Access With a Novel Asynchronous Interference Cancellation Technique," IEEE Transactions on Communications,vol. 65 , no. 3 , March 2017.

[19] J. Ghosh, D. Das, "Femtocells Underlaid Macrocellular Networks," IETE Technical Review, Nov 2019. DOI:10.1080/02564602.2019.1682693.

[20] H. O. Kpojime, G. A. Safdar, "Interference Mitigation in CognitiveRadio-Based Femtocells," IEEE Communications Surveys and Tutorials, vol. 17, no. 3, pp.1511 - 1534, 2015.

[21] A. Gupta, R. K. Jha,"A Survey of 5G Network: Architecture and Emerging Technologies,” IEEE Access, vol.3, pp.1206 - 1232, July 2015.

[22] T. D. Novlan, R. K. Ganti, A. Ghosh, J. G. Andrews, "Analytical Evaluation of Fractional Frequency Reuse for Heterogeneous Cellular Networks," IEEE Trans. Commun., vol.60, no.7, July 2012.

[23] L. Liang, G. Feng, T. Mao, "Interference coordination based on access control in macro-femto networks,'IEEE Wireless Communications and Networking Conference (WCNC), Shanghai, pp. 2277-2282, 2013.

[24] B. Niu, V. W. S. Wong, "Network Configuration for Two-Tier Macro-Femto Systems With Hybrid Access," IEEE Transactions on Vehicular Technology, vol. 65, no. 4, pp. 2528-2543, April 2016. 
[25] A. Pratap, R. Singhal, R. Misra, S. K. Das, "Distributed Randomized k-Clustering based PCID Assignment for Ultra-Dense Femtocellular Networks," IEEE Trans. on Parallel and Distributed Systems, vol. 29, no.6, pp.1247-1260, June 2018.

[26] N. Ul Hasan, W. Ejaz, N. Ejaz, H. S. Kim, A. Anpalagan and M. Jo "Network Selection and Channel Allocation for Spectrum Sharing in 5G Heterogeneous Networks," IEEE Access, vol.4, pp.980-992, 2016.

[27] Z. Pan, S. Shimamoto, "Cell sizing based energy optimization in joint macro-femto deployments via sleep activation," IEEE Wireless Communications and Networking Conference (WCNC), Shanghai, pp. 4765-4770, 2013.

[28] Z. Liu, L. Gao, Y. Liu, X. Guan, K. Ma and Y. Wang, "Efficient QoS Support for Robust Resource Allocation in Blockchain-Based Femtocell Networks," IEEE Transactions on Industrial Informatics, vol. 16, no. 11, pp. 7070-7080, Nov. 2020

[29] H. Ghazzai, M. J. Farooq, A. Alsharoa, E. Yaacoub, A. Kadri, MS Alouini, "Green Networking in Cellular HetNets: A Unified Radio Resource Management Framework with Base Station ON/OFF Switching,'IEEE Transactions on Vehicular Technology, 2017.

[30] J. Tang, D. So, E. Alsusa, K. Hamdi, A. Shojaeifard, "Resource allo- cation for energy efficiency optimization in heterogeneous networks," IEEE J. Sel. Areas Commun., pp. 2104-2117, Oct. 2015.

[31] A. R. Elsherif, W. Chen, A. Ito, Z. Ding, "Adaptive Resource Allocation for Interference Management in Small Cell Networks," IEEE Transactions on Communications, vol. 63, no. 6, pp. 2107-2125, June 2015.

[32] M. Azmy, K. ElAzzouni, A. Abuemeira, M. ElNainay, "A Genetic Algorithm-Based Approach for Content Delivery in FemtocachingAssisted Networks," IEEE Wireless Communications and Networking Conference (WCNC), San Francisco, CA, USA, May 2017.

[33] A. Kumbhar, I. Güvenç, S. Singh, A. Tuncer, "Exploiting LTE-Advanced HetNets and FeICIC for UAV-Assisted Public Safety Communications," IEEE Access Feb. 2018.

[34] M. Haenggi, R. K. Ganti, "Interference in Large Wireless Networks," Foundations and Trends in Networking, vol. 3, no.2, pp.127-248, 2008

[35] https://portal.3gpp.org/desktopmodules/Specifications /SpecificationDetails.aspx? specificationId=319.

[36] N. Kong, L. B. Milstein,"Average SNR of a Generalized Diversity Selection Combining Scheme,'IEEE Communications Letters, Vol. 3, no. 3, March 1999. 